\title{
Metastatic Squamous Cell Carcinoma of the Lower Lip: Analysis of the 5-Year Survival Rate
}

\author{
Tommaso Agostini ${ }^{1,2}$, \\ Giuseppe Spinelli, \\ Francesco Arcuri ${ }^{1}$, \\ Raffaella Perello ${ }^{2}$ \\ ${ }^{1}$ Department of Maxillo-Facial Surgery, CTO- \\ AOUC, University of Florence, Florence; \\ ${ }^{2}$ Department of Plastic and Reconstructive \\ Surgery, Centro Chirurgico San Paolo, Pistoia, \\ Italy
}

No potential conflict of interest relevant to this article was reported.

\begin{abstract}
Background: The author analyse the impact of extracapsular lymph node spread and bone engagement in the ipsilateral neck of patients suffering squamous cell carcinoma (SCC) of the lower lip.

Methods: The data of 56 neck dissections performed in patients suffering SCC of the lower lip between January 2000 and December 2008 were retrospectively analysed. Statistical analysis was performed with the Kaplan-Meier life table method, and the survival rate was investigated with the log rank statistic and significance test. The values were considered statistically significant at $p<0.05$.

Results: Nine patients took advantage from simultaneous treatment of tumor and prophylactic neck dissection (level I-III), reaching 100\% survival rate. Patients suffering metastasized disease, who received radical neck dissection at the time of tumor treatment, presented $83.3 \%$ survival rate. Patients who underwent previous surgery and radiotherapy presented worse prognosis although radical neck dissection in case of extra-capsular spread only (24.7\%) and osseous engagement (22.2\%).

Conclusion: Prophylactic neck dissection (level I-III) is recommended in T3-T4 N0 SCC. Simultaneous treatment of tumor and cervical lymph nodes provides a better prognosis as respect to delayed nodal management. Extra-capsular spread with or without bone engagement represents independent risk factor responsible for high mortality rate of SCC of the lower lip.
\end{abstract}

Keywords: Squamous cell carcinoma / Lip / Neck dissection

\section{INTRODUCTION}

Tumours of the lip are relatively common lesions and are not adequately acknowledged as potentially fatal. Squamous cell carcinoma (SCC) is a true invasive carcinoma of the surface epidermidis. It affects patients over 50 year-old with fair skin, blonde hair and blue eyes [1-4]. Contribute factors include sola damage, radiation, chronic wounds, human papilloma virus, chemical carcinogens, immunosuppression, inflammatory disorders and genetic syndromes. SCC may arise de novo in predisposed pa-

Correspondence: Tommaso Agostini

Department of Maxillo-Facial Surgery, University of Florence, Largo Palagi 1, Florence, Italy E-mail: tommasoagostini@ymail.com

Received March 28, 2017 / Revised June 19, 2017 / Accepted June 20, 2017 tients or from precursor lesions [4,5]. Well-controlled surgical excision of primary SCC achieves 5 years cure rate of $92 \%$ with overall recurrence rate of $8 \%$ [6]. Several factors may influence a misdiagnosis: low incidence of lymph node metastasis, slow diffusion, deep metastasis, lymph node jump, fixation of lymph node metastasis to mandibular periosteum, and previous radiotherapy. Several factors are directly correlated to lymph node involvement, including tumour size and differentiation. Orbicularis oris invasion incidence has been reported to be as high as $18.3 \%[4-8]$.

The aim of this study was to analyse lower lip tumours that metastasised to the cervical lymph nodes and to identify the prognostic factors of a poor prognosis. To collect a uniform cohort of patients, we focused the present retrospective analysis on identify- 
ing patients suffering from SCC of the lower lip that was metastatic at the time of surgery or that metastasised a second time following the first treatment.

\section{METHODS}

A retrospective chart review was conducted in the Department of Maxillo Facial Surgery of the University of Florence between January 2000 and December 2008. The clinical records of patients suffering metastasised SCC of the lower lip were reviewed, and autopsy and death certificates were reviewed to identify the cause of death. During the eight years reviewed, 56 neck lymph node dissections were performed following metastasised SCC of the lower lip. The patients' age ranged from 45 to 87 years, and the mean age was 65.9 years. The patients were $91 \%$ male and $9 \%$ female.

Patients suffering T1 and T2 tumours underwent excision and immediate reconstruction, and patients suffering T3-T4 tumours underwent preoperative incisional biopsy of the primary lesion. Surgical treatment consisted of 1-cm healthy looking tissue resection, and in the identified cohort, all of the definitive histological margins were free from disease. In particular, surgical margins were intraoperatively checked using frozen sections for T3-T4 tumours but never checked in cases with smaller lesions. Prophylactic selective neck dissection was deserved to patients suffering T3 and T4 SCC of the lower lip.

Ethical approval was performed by the local committee of the Azienda Ospedaliero Universitaria Careggi (reference number OSS_14.136).

\section{Statistical analysis}

Retrospective analysis of the collected data was performed using the Kaplan-Meier life table method, and the survival rate was investigated with the log rank statistic and significance test. The data were analysed with SPSS ver. 10.0 for Windows (SPSS Inc., Cary, NC, USA), and the values were considered statistically significant at $p<0.05$.

\section{RESULTS}

Nine patients (node negative) $(16 \%, \mathrm{n}=9 / 56)$ underwent prophylactic selective neck dissection (level I to III) at the time of primary tumor resection; 47 patients (node positive) $(84 \%, n=47 / 56)$ underwent radical neck dissection: level Ib (submandibular) was the most involved, accounting for 36 patients $(76.6 \%, \mathrm{n}=36 / 47)$, and 16 of these patients $(44.4 \%, \mathrm{n}=16 / 36)$ suffered lymph node metastasis that was adherent to the mandible (Fig. 1). Three patients (6.3\%, $\mathrm{n}=3 / 47$ ) suffered lymph node metastasis at level Ia (submental), with only one adherence to the mandible $(33.3 \%, n=1 / 3)$. One patient $(2.1 \%, \mathrm{n}=1 / 47)$ suffered metastasis to Level II and 2 patients to Level III (4.2\%, n=2/47) (Table 1).

Then, we divided the 47-patient cohort that underwent neck dissection into two groups: group 1, radical neck dissection performed at the time of tumour resection and immediate reconstruction $(\mathrm{n}=6)$; and group 2, neck dissection of patients following surgery and radiotherapy who referred to our department from other hospitals ( $\mathrm{n}=41)$ (Fig. 1). This last group was further divided according to the adherence of lymph node metastasis to the mandible: subgroup $2 \mathrm{~A}$ without adherence to the mandible and subgroup $2 \mathrm{~B}$ with nodal adherence to the mandible. Subgroup 2A, including patients without adherence to the mandible, were further classified according to the soft tissue extra-capsular spread only: subgroup 2C (without extracapsular spread) $(\mathrm{n}=17)$ and subgroup 2D (with extravascular spread) (n=9) (Fig. 1). Then, we analysed the divided cohort groups to identify the poor prognostic factors responsible for the high disease mortality rate observed in the study.

We did not record complications following neck dissection, such as haemorrhage, infection, flap necrosis, fistula or functional damage to noble structures. Furthermore, we also did not retrieve the parotid lymph node data in this study in cases of commissural tumours. Nine patients $(16 \%, n=9 / 56)$ who underwent prophylactic neck dissection were alive at the 5-year follow-up (Table 2), and neck dissection was performed following a final histological diagnosis in high-risk patients (poorly differentiated tumours). Group 1 (tumor resection and neck dissection): six patients $(2.8 \%$, $\mathrm{n}=6 / 47$ ) suffering T2-T3-T4 tumours and lymph node metastasis 


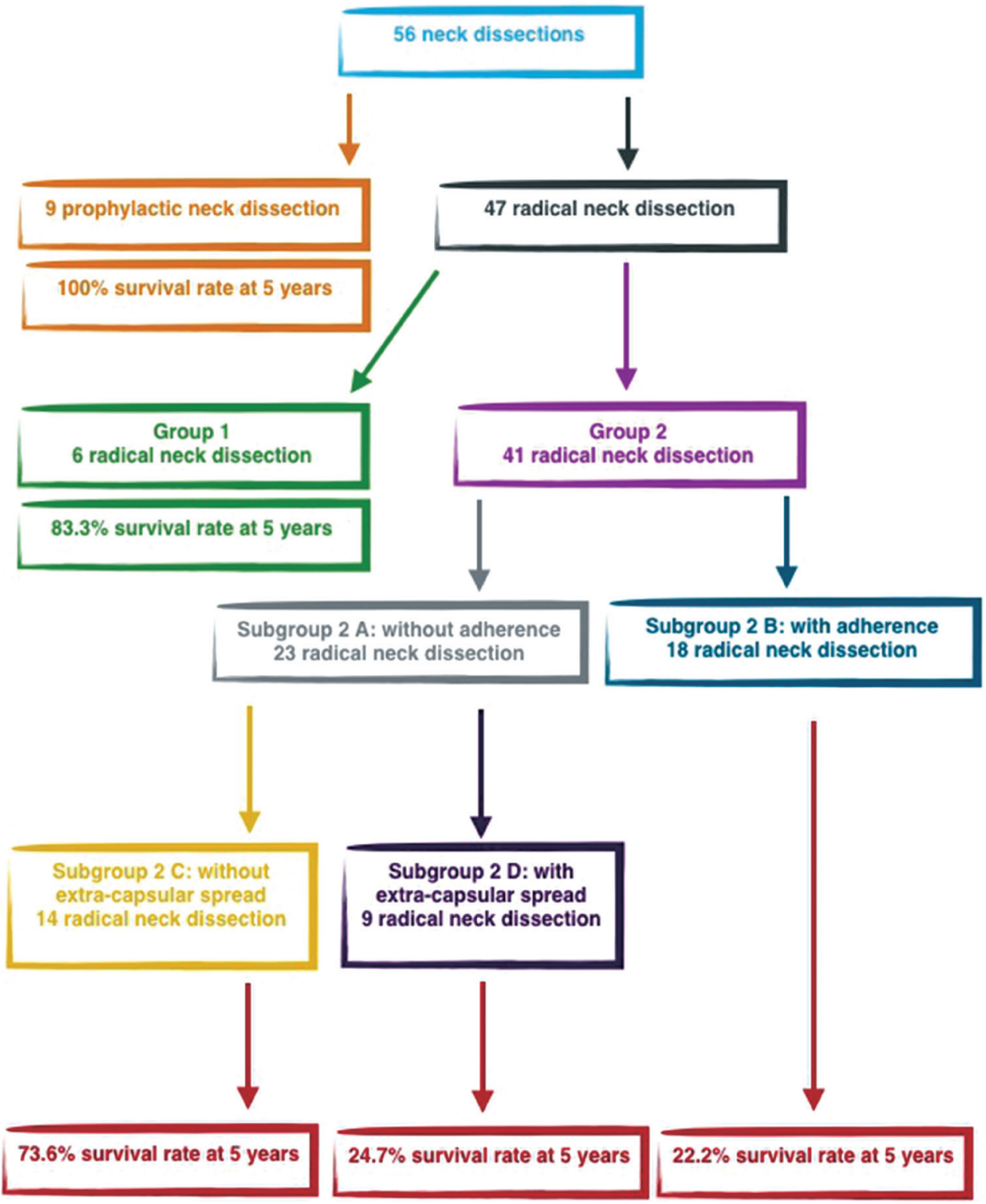

Fig. 1. Schematic representation of the 56 neck dissection performed and the 5-year survival rate analysis.

Table 1. Distribution of lymph node metastasis in patients $\mathrm{N}+$ suffering squamous cell carcinoma of the lower lip $(n=47)$

\begin{tabular}{lcc} 
Neck level & Total N involved (\%) & $\begin{array}{c}\text { N with adherent } \\
\text { metastasis (\%) }\end{array}$ \\
\hline la & $8(17)$ & $3(37.5)$ \\
Ib & $36(76.6)$ & $16(44.4)$ \\
II & $1(2.1)$ & - \\
III & $2(4.2)$ & - \\
\hline
\end{tabular}

N, lymph node.
Table 2. Data of patients undergone prophylactic selective neck dissection $(n=9)$

\begin{tabular}{lcl}
\hline TNM & No. of patients (\%) & Type of neck dissection \\
\hline T2NO & 2 & Level I-III \\
\hline T3NO & 1 & Level I-III \\
\hline Recurrences & 5 & Level I-III (bilaterally) \\
\hline Recurrences & 1 & Level I-V \\
\hline
\end{tabular}

All patients (100\%) were alive at 5-year follow-up. 
underwent radical neck dissection at the time of tumour excision and reconstruction (Table 3). Of these patients, only 1 patient (T3N2b; 16.6\%, n=1/6) suffered an adherent mandibular lymph node metastasis and died of the disease by the 5-year follow-up. Group 2 (neck dissection following surgery and adjuvant therapy): forty-one patients $(87.2 \%, \mathrm{n}=41 / 47)$ underwent a radical neck dissection and were previously treated with surgery and radiotherapy in other hospitals. This group was divided according to the adherence of the metastasis to the mandibular periosteum. Subgroup $2 \mathrm{~A}$ included 23 patients $(56 \%, \mathrm{n}=23 / 41$ ) suffering nonadherent lymph node metastasis (14 N1 patients and 9 N2a patients) and subgroup 2B with 18 patients ( $43 \%, \mathrm{n}=18 / 41)$ suffering adherence to the mandible (10 N2b patients and $8 \mathrm{~N} 3$ patients). Ten patients (71.4\%, $\mathrm{n}=10 / 14$ ) of subgroup 2C (without extracapsular spread) were alive at 5 years follow-up and 2 patients (22.2\%, $\mathrm{n}=2 / 9$ ) of subgroup $2 \mathrm{D}$ (with extravascular spread) were alive at 5 years follow-up. Of the 18 patients suffering adherent lymph node metastasis (subgroup $2 \mathrm{~B}$ ), only 4 patients (N2a) $(22.2 \%, \mathrm{n}=4 / 18)$ were free from the disease at the 5-year follow-up following extended radical neck dissection. Fourteen patients $(10 \mathrm{~N} 2 \mathrm{~b}$ patients and $4 \mathrm{~N} 3$ patients) $(77.7 \%, \mathrm{n}=14 / 18)$ died of the disease ( 2 of them with lung metastasis) (Table 4) (Fig. 1). According to the retrospective review, a total of 19 patients (33.9\%, $\mathrm{n}=19 / 56)$ presented with

Table 3. Patients undergone neck dissection at the time of tumor excision and reconstruction $(\mathrm{n}=6)$

\begin{tabular}{lll}
\hline TNM $($ no.,$\%)$ & Type of neck dissection & Status \\
\hline T2N1 $(1,16)$ & Radical neck dissection & Alive \\
\hline T4N1 $(2,33)$ & Radical neck dissection & Died \\
\hline T3N2a $(1,16)$ & Radical neck dissection & Alive \\
\hline T3N2b $(2,33)$ & Radical neck dissection & 1 Alive \\
\hline
\end{tabular}

Three patients (50\%) were alive at the time of 5-year follow-up. Only the patient died in the T3N2c group suffered metastasis adherence to the mandibular periosteum.

Table 4. Neck dissection in patients with recurrence of $\mathrm{N}$ following previous surgery and radiotherapy $(\mathrm{n}=41)$

\begin{tabular}{lclcc} 
N (lymph node) & No. & N involvement & Alive & Died (\%) \\
\hline N non adherent & 23 & $\begin{array}{l}14 \mathrm{~N} 1 \\
9 \mathrm{~N} 2 \mathrm{a}\end{array}$ & 21 & $2(8.6)$ \\
N adherent & 18 & $\begin{array}{l}10 \mathrm{~N} 2 \mathrm{~b} \\
8 \mathrm{N3}\end{array}$ & $4(\mathrm{~N} 3)$ & $14(77.7)$ \\
\hline
\end{tabular}

Fifteen patients (79\%) suffering mandibular adherence died of disease at 5-year follow-up.

$\mathrm{N}$, lymph node. adherence of the metastasis to the mandible as the expression of a malignant extra-capsular breakdown with bone engagement and 6 patients $(10.7 \%, \mathrm{n}=6 / 56)$ suffered extracapsular spread only. Eighteen patients received previous treatment in other hospitals (primary tumor surgery), and only 1 patient had never received previous treatments. Twenty-six patients of the 47 radical neck dissection performed $(55.3 \%, \mathrm{n}=26 / 47)$ died of the disease by the 5 -year follow-up, which validates that both adherence to the mandible and extra-capsular spread are index of high mortality in lower lip SCC, and these indexes are independent of aggressive surgical management combined or not with radiotherapy $(p<0.05)$. Forty-seven patients of the cohort $(84 \%, \mathrm{n}=47 / 56)$ underwent combined radiotherapy and chemotherapy.

\section{Overall timing of onset of mandibular adher- ence $(n=56)$}

Four patients $(7.1 \%, \mathrm{n}=4 / 56)$ developed adherence within 3 years of treatment, and 15 patients $(26.8 \%, \mathrm{n}=15 / 56)$ suffered adherence during the last two years of the 5 -year follow-up $(p<0.05)$.

\section{Specific analysis of group 2 (41 patients)}

This group of patients counts 41 patients referring to our Department from other Hospitals and suffering clinical nodal metastasis adherent to the mandible. Initial TNM stage was available in only 5 cases while TNM stage at presentation is available in Table 4 . Eighteen patients were free from local recurrence $(0 \%, \mathrm{n}=0 / 41)$ and 23 patients ( $56 \%, \mathrm{n}=23 / 41)$ suffered local recurrences distributed as follow: 12 patients with T3 SCC and 11 patients with T4 SCC. This group of patients is divided into two subgroup: subgroup A counting 23 patients (56\%, $n=23 / 41$ ) suffering non-adherent lymph node metastasis and subgroup B with 18 patients $(43 \%, \mathrm{n}=18 / 41)$ with adherence to the mandible. Between the subgroup A, 9 patients $(39.1 \%, n=9 / 23)$ presented soft tissue extracapsular spread only, and 2 of them $(22.2 \%, \mathrm{n}=2 / 9)$ were alive at five years follow-up (Fig. 1).

All of them underwent extended radical neck dissection and close clinical observation of the contralateral neck. Mandibular 
involvement was evaluated in the preoperative with Computed Tomography and it was faced with marginal resection of the lower border with no radiographic evidence of bone invasion and with segmental resection in case of bone erosion. Oromandibular reconstruction was performed with pectoralis major myocutaneous flap and a metal reconstruction plate. The reconstructive safety was enhanced by rolling the pectoralis major myocutaneous flap around the metal plate, to minimize the amount of the primary reconstruction time in this cohort of patients with a poor prognosis. This type of reconstruction gives further advantages of preserving both the alloplastic material and the remaining noble structures in omolateral depleted neck (carotid artery and vagus nerve) from the detriment effect of postoperative adjuvant therapy. Patients who underwent reconstruction, well-tolerated radiotherapy without complications.

\section{DISCUSSION}

The lips are a common and often overlooked site for non-melanoma skin cancers (NMSC), including two frequent carcinomas: basal and squamous cell carcinoma (BCC and SCC) [9-12]. These carcinomas have a tendency to occur in fair-skinned males over 50 years of age. Males are up to 13 times more likely to develop lip cancers $[13,14]$. The lower lip is 12 times more likely to be affected because of its greater exposure to sunlight. Given their high visibility, the majority of lesions are easily detectable and curable at an early stage. Recurrence ranges from 5\% to 35\%, and the mortality associated with large or recurrent skin cancer reaches 15\% in some studies [15-19]. In cases of local lymph node involvement, the fiveyear mortality is approximately $50 \%[3,5,7,12,20-23]$. Recurrence rate varies according to size, location, previous treatment and histology. Radiotherapy is indicated, beyond inoperable tumors, for SCC arising in high-risk area (face), multiple lesions and nodal involvement [2]. Dose range from 4,000 to 6,000 CGy in multiple fractions of 200-300 cGy each and with $2 \mathrm{~cm}$ margins [5]. Tumour control in patients with cervical metastasis is considerably more difficult. The overall curability of patients with metastasised lower lip tumours approaches 50\% [1-3]. The incidence of lip tumour metastasis to cervical lymph nodes ranges from $5 \%$ to $20 \%$, with level I metastases being the most common [4].

Although, primary SCC metastasizes in $0.3 \%-0.6 \%$ of cases, tumor's spread tends to increase when it arises on the lips (11\%), ear (14\%), chronic wounds (40\%-50\%) and in case of recurrence $(23 \%-30 \%)$ [13-17]. The risk of metastasis increases for tumors larger than $2 \mathrm{~cm}$ and deeper than $4 \mathrm{~cm}$, recurrent tumors, bone invasion, perineural and perivascular involvement [13]. Approximately $95 \%$ of metastases are detected within 2 years and the reported 5-year survival for metastatic SCC is 25\% [2,7-9,12-15]. Neck management in patients suffering invasive SCC depends of the presence/absence of clinical nodal disease. Although for patients without evident nodal involvement (N0), close observation is reasonable, tumors with a $20 \%$ chance of metastases (large, undifferentiated, recurrent) require suprahyoid neck dissection (level I to III) and close observation of level IV and V. In case of positive lymph nodes, a modified radical neck dissection is recommended. In case of clinical nodal involvement a radical neck dissection is indicated $[3,4,7,14,19,21,24]$.

Some centres do not recommend prophylactic elective neck dissection because of the low occult metastasis rate of lower lip carcinoma and these centres reserve modified radical neck dissection for patients developing cervical metastasis later $[4,7,9,14,24,25]$. However, prophylactic neck dissection is the only method to detect occult metastasis. This topic is hotly debated. According to Jones et al. [25], elective neck dissection has a minimal effect on survival rate, whereas, in contrast, Onercl et al. [16] did not record metastasis in their N0 patients who had undergone elective neck dissection and concluded that elective neck dissection provides superior disease control in respect to a wait-and-see approach [4,5,7,11,12,14,17-24].

The risk of metastatic spread is greater for lesions larger than 2 $\mathrm{cm}$ or deeper than $4 \mathrm{~mm}$ or those that have recurrences, bone invasion, and perineural involvement. For patients without clinically evident nodes, a 3-month close observation is reasonable $[4,7,8,12-23]$. However, tumours with at least a $20 \%$ chance of metastasis, such as large, undifferentiated and recurrent tumours, require neck dissection and treatment of the primary site [16-22]. Suprahyoid neck dissection results in reduced morbidity related to radiotherapy and radical neck dissection. This surgical proce- 
dure removes nodal levels I (submandibular), II (high cervical) and III (midcervical) $[18,19]$. If the nodes removed are negative, then observation is recommended because of the possibility of positive nodes in level IV and $\mathrm{V}$ is very low. In positive cases, modified neck dissection is indicated $[15,18,22]$. Radiotherapy should be added if further examination reveals multiple node metastasis or extracapsular invasion [25].

Lower lip SCC displays a low tendency to metastasise to cervical lymph nodes, with a frequency ranging from 5\% to 20\% [2]. Submental and submandibular lymph nodes (level I) display the earliest involvement as a basis for tumour advancement, unlike other later involved cervical bases. The poor tendency to give metastasis, relatively low incidence of metastasis and slow tumour advancement should not result in an underestimation of tumour evolution, especially in cases of lymph node basis jumping or adherence to the mandibular periosteum $[7,14,21,22]$. In particular, clinical examination can be very difficult for experts as well in cases with patients who have undergone radiotherapy and have hardened neck soft tissue, which may mask deep or adherent lymph node metastasis [11,24]. Several factors influence metastasis development, including tumour size and differentiation [1]. With regards to the first parameter, in the case of T1 tumours, lymph node engagement ranges from $3.4 \%$ to $7 \%$. For T2 tumours, engagement ranges from $11.2 \%$ to $35 \%$, and for T3 tumours, engagement ranges from $26.4 \%$ to $63 \%$. Similarly, well-differentiated tumours are characterised by a 7\% incidence of lymphnode metastasis compared with the $21 \%$ in the case of nondifferentiated tumors $[4,10,11,24]$.

In accordance with the medical literature, the distribution of lymph node metastasis in the present series mainly involved level I [2,4,7,12-15,24,25]. Lymph node involvement represents the most critical index of patient survival in the 5-year follow-up, with a mortality ranging from $50 \%$ up to $70 \%$ in cases of adherence to the mandible $[4,7,14]$. The low survival rate linked to lymph-node adherence is the result of capsular breakdown with osseous engagement, perivascular and perineural invasion $[5,8]$. The results of the present study indicate that 9 patients took advantage from simultaneous treatment of tumor and prophylactic neck dissection level I-III, reaching 100\% survival rate at 5-year follow-up even in case of advanced SCC (T2-T3) or recurrences. Patients who underwent radical neck dissection following lymph node metastasis presented lower survival rate at 5-year follow-up. In particular, level I lymph node metastasis was distributed on a submental basis in 8 patients (17\%) with a $37.5 \%$ incidence of adherence to the mandible and submandibular in 36 cases (76.6\%) with $44.4 \%$ nodal adherence to the mandible. Patients suffering metastasized disease who received radical neck dissection at the time of tumor treatment (group 1) presented 83.3\% survival rate at 5-year follow-up. Patients who underwent previous surgery and radiotherapy presented worse prognosis although radical neck dissection in case of extra-capsular spread only (subgroup 2D, 24.7\% survival rate at 5-year follow-up) and osseous engagement (subgroup 2B, 22.2\% survival rate at 5-year follow-up). Patients suffering modal metastasis without extra-capsular spread had a better survival rate of $73.6 \%$ at 5 -year follow-up.

Early detection and treatment of lower lip tumours has a quite favourable cure rate beyond 90\%, with surgery or radiotherapy alone. However, the 5-year survival rate for patients with cervical metastasis is reported to be between $30 \%$ and $70 \%$, with an average of 50\% [12,14,23]. Twenty-six patients of the 47 radical neck dissection performed (55.3\%) died of the disease by the 5-year follow-up and these data are in accordance with the medical literature $[12,24,25]$. However specific analyses of subgroups identified two independent rick factors responsible for a prognosis: mandible engagement and extra-capsular spread only.

Moreover, in this retrospective review, we found that mandibular adherent metastasis was detected during the last 2 years of the canonical follow-up, and it can be ascribed to the subgroup of the patients who underwent previously surgery combined with radiotherapy, thus making clinical and radiographic detection more difficult. We conclude that we should be treating the neck prophylactically more often, and we should be attempting to identify high-risk patients based on lip pathology, sentinel node biopsy or neck dissection. In cases of suspension, we strongly encourage immediate prophylactic suprahyoid neck dissection as a biopsy tool, followed by radical neck dissection if indicated. In particular, prophylactic neck dissection is useful for T3-T4 tumours, highgrade tumours, in patients who may not return for clinical follow- 
up and, finally, in the case of recurrence. Physicians should be following patients more closely and educating practitioners and patients regarding the risks of neck metastases, along with the proper treatment.

Surgical management of patients suffering T1-T2 N0 lower lip SCC remains a matter of debate, and selective neck dissection is not recommended. The authors prefer a short-term three-month follow-up (clinical examination conjoined to bilateral cervical ultrasonography) for the early detection of lymph node metastasis to prevent capsular breakdown and periosteal adherence [1-5]. Prophylactic neck dissection (I-III) is recommended in the case of T3-T4 N0 SCC. Simultaneous treatment of tumor and cervical lymph nodes provides a better prognosis as respect to delayed nodal management. Extra-capsular spread with or without bone engagement represents independent risk factor responsible for high mortality rate of SSC of the lower lip.

\section{REFERENCES}

1. Takeda A, Akimoto M, Nemoto M, Kounoike N, Uchinuma E. Preoperative risk factors of lymph node metastasis in cutaneous squamous cell carcinoma. J Plast Surg Hand Surg 2013;47:204-8.

2. Dediol E, Luksic I, Virag M. Treatment of squamous cell carcinoma of the lip. Coll Antropol 2008;32 Suppl 2:199-202.

3. Morselli P, Masciotra L, Pinto V, Zollino I, Brunelli G, Carinci F. Clinical parameters in T1N0M0 lower lip squamous cell carcinoma. J Craniofac Surg 2007;18:1079-82.

4. Gooris PJ, Vermey A, de Visscher JG, Burlage FR, Roodenburg JL. Supraomohyoid neck dissection in the management of cervical lymph node metastases of squamous cell carcinoma of the lower lip. Head Neck 2002;24:678-83.

5. Vukadinovic M, Jezdic Z, Petrovic M, Medenica LM, Lens M. Surgical management of squamous cell carcinoma of the lip: analysis of a 10-year experience in 223 patients. J Oral Maxillofac Surg 2007; 65:675-9.

6. Kornevs E, Skagers A, Tars J, Bigestans A, Lauskis G, Libermanis O. 5 year experience with lower lip cancer. Stomatologija 2005;7:95-8.

7. Dunne AA, Budach VG, Wagner W, Werner JA. Management of N0 neck in head and neck cancer: current controversies. Onkologie 2004; 27:363-7.

8. Bucur A, Stefanescu L. Management of patients with squamous cell carcinoma of the lower lip and N0-neck. JCraniomaxillofac Surg 2004; 32:16-8.

9. de Visscher JG, van den Elsaker K, Grond AJ, van der Wal JE, van der Waal I. Surgical treatment of squamous cell carcinoma of the lower lip: evaluation of long-term results and prognostic factors--a retrospective analysis of 184 patients. J Oral Maxillofac Surg 1998;56:81420 .

10. Rodolico V, Barresi E, Di Lorenzo R, Leonardi V, Napoli P, Rappa F, et al. Lymph node metastasis in lower lip squamous cell carcinoma in relation to tumour size, histologic variables and p27Kip1 protein expression. Oral Oncol 2004;40:92-8.

11. Kutluhan A, Kiris M, Kaya Z, Kisli E, Yurttas V, Icli M, et al. Squamous cell carcinoma of the lower lip and supra-omohyoid neck dissection. Acta Chir Belg 2003;103:304-8.

12. Bilkay U, Kerem H, Ozek C, Gundogan H, Guner U, Gurler T, et al. Management of lower lip cancer: a retrospective analysis of 118 patients and review of the literature. Ann Plast Surg 2003;50:43-50.

13. Kocaturk S, Ozdemir N, Erkam U, Uzun H, Babila A, Ozturk E. Evaluation of occult lymph node metastasis in lower lip cancers and approach to N(0) neck metastasis. Kulak Burun Bogaz Ihtis Derg 2002; 9:41-5.

14. Altinyollar $\mathrm{H}$, Bulut $\mathrm{H}$, Berberoglu U. Is suprahyoid dissection a diagnostic operation in lower lip carcinoma? J Exp Clin Cancer Res 2002;21:29-30.

15. Veness MJ, Ong C, Cakir B, Morgan G. Squamous cell carcinoma of the lip. Patterns of relapse and outcome: Reporting the Westmead Hospital experience, 1980-1997. Australas Radiol 2001;45:195-9.

16. Onercl M, Yilmaz T, Gedikoglu G. Tumor thickness as a predictor of cervical lymph node metastasis in squamous cell carcinoma of the lower lip. Otolaryngol Head Neck Surg 2000;122:139-42.

17. de Visscher JG, Botke G, Schakenraad JA, van der Waal I. A comparison of results after radiotherapy and surgery for stage I squamous cell carcinoma of the lower lip. Head Neck 1999;21:526-30.

18. Daniele E, Rodolico V, Leonardi V, Tralongo V. Prognosis in lower lip squamous cell carcinoma: assessment of tumor factors. Pathol Res Pract 1998;194:319-24.

19. Koc C, Akyol MU, Celikkanat S, Cekic A, Ozdem C. Role of suprahyoid neck dissection in the treatment of squamous cell carcinoma of the lower lip. Ann Otol Rhinol Laryngol 1997;106:787-9.

20. Zitsch RP 3rd, Park CW, Renner GJ, Rea JL. Outcome analysis for lip carcinoma. Otolaryngol Head Neck Surg 1995;113:589-96.

21. Liang XH, He YW, Tang YL, Wu JL, Cao XP, Xiao GZ, et al. Thermochemotherapy of lower lip squamous cell carcinoma without metastases: an experience of 31 cases. J Craniomaxillofac Surg 2010;38:260-5.

22. Hjortdal O, Naess A, Berner A. Squamous cell carcinomas of the lower lip. JCraniomaxillofac Surg 1995;23:34-7.

23. Bagatin M, Orihovac Z, Mohammed AM. Perineural invasion by carcinoma of the lower lip. J Craniomaxillofac Surg 1995;23:155-9.

24. Guney E, Yigitbasi OG. Functional surgical approach to the level I for staging early carcinoma of the lower lip. Otolaryngol Head Neck Surg 2004;131:503-8.

25. Jones AS, Phillips DE, Helliwell TR, Roland NJ. Occult node metastases in head and neck squamous carcinoma. Eur Arch Otorhinolaryngol 1993;250:446-9. 